\title{
Symmetry-Preserving Difference Models of Some High-Order Nonlinear Integrable Equations
}

\section{Zhonglong Zhao ${ }^{1}$}

Received: 7 April 2021 / Accepted: 3 August 2021 / Published online: 20 September 2021

(C) The Author(s) 2021

\begin{abstract}
In this paper, a procedure for constructing the symmetry-preserving difference models by means of the potential systems is employed to investigate some kinds of integrable equations. The invariant difference models for the Benjamin-Ono equation and the nonlinear dispersive $K(m, n)$ equation are investigated. Four cases of $K(m, n)$ equations which yield compactons are studied. The invariant difference models preserving all the symmetries are obtained. Furthermore, some linear combinations of the symmetries are used to construct the invariant difference models. The invariant difference model of the Hunter-Saxton equation is constructed. The idea of this paper can be further extended to discrete some other high-order nonlinear integrable equations.
\end{abstract}

Keywords Symmetry-preserving · Difference models · Benjamin-Ono equation · Nonlinear dispersive $K(m, n)$ equation · Hunter-Saxton equation

Mathematics Subject Classification 58D19 · 58J70 - 65Q10

\section{Introduction}

Lie symmetry analysis method is one of the most effective method to study the properties of partial differential equations [1-7]. One of the main application of Lie symmetry is to find the group invariant solutions [8-10]. The structure of the Lie algebra spanned by Lie symmetry operators provides important information about the set of the solutions. High dimensional transformation group (HDTG) may have more possible applications. Therefore, for a finite-difference model, it is an important property to preserve the symmetries of its original continuous model.

Zhonglong Zhao

zhaozlhit@163.com; zhaozl@nuc.edu.cn

1 Department of Mathematics, North University of China, Taiyuan 030051, Shanxi,

People's Republic of China 
Compared with the study of the symmetry method to the continuous equation, the symmetry study of the difference equation is relatively less. In the past few decades, some scholars tried to solve some problems of difference equation with symmetry method. Maeda [11] investigated the analytic solutions of two nonlinear difference equations by means of the similarity method. Levi and Winternitz [12] extended the concept of conditional symmetry to the differential-difference equations. Shen et al. $[13,14]$ discussed the symmetry classifications of some differential-difference equations. A systematic method was proposed to derive nonlocal symmetries for the differential-difference equations [15]. The structure of the symmetry group palys an important role in constructing the difference equation and grides. Group transformations can destroy the geometric structure of the mesh and affect the properties of lattice equation. Dorodnitsyn et al. [16-18] systematically studied the problem of constructing the difference model which preserves the symmetries of original continuous equation. It's worth noting that a method of constructing symmetrypreserving difference scheme by means of potential systems for original continuous equation was proposed by Xin and Chen [19, 20]. Then Tian et al. [21, 22] obtained invariant difference schemes of some (1+1)-dimensional and high-dimensional nonlinear differential equations.

The organization of the paper is as follows. In Sect. 2, we briefly present the procedure for constructing the symmetry-preserving difference scheme of partial differential equations by means of the potential systems. In Sect. 4, the invariant difference model of the Benjamin-Ono equation is obtained. We prove that the invariant difference model is invariant under the action of extension symmetry. In Sect. 5, the nonlinear dispersive $K(m, n)$ equations which admit compactons are studied to construct invariant difference models. In Sect. 6, we use the linear combinations of some symmetries to construct the invariant difference models, which preserve some part of symmetries of original equation. The invariant difference model of the Hunter-Saxton equation is investigated as an example. Finally some conclusions are given in the last section.

\section{Preliminaries for Construction of Invariant Difference Models}

In this section, we briefly introduce the bases of the finite-difference derivatives and a procedure for constructing the symmetry-preserving difference scheme. For the details, the readers are referred the references [19, 23]. Let us consider the space of sequences $\left(x, u, u_{1}, u_{2}, \ldots\right)$, where independent variable is $x=\left\{x^{i} ; i=1,2, \ldots, n\right\}$, dependent variable is $u=\left\{u^{k} ; k=1,2, \ldots, m\right\}, u_{1}=\left\{u_{i}^{k}\right\}$ represents the set of $m n$ first derivatives, $u_{2}=\left\{u_{i j}^{k}\right\}$ is the set of second order partial derivatives, etc. In order to briefly introduce the finite-difference derivatives, we consider the simple case $n=2$, i.e., $x=\left(x_{1}, x_{2}\right)$.

The total derivative operators are introduced by 


$$
\begin{aligned}
& D_{1}=\frac{\partial}{\partial x^{1}}+u_{1} \frac{\partial}{\partial u}+u_{11} \frac{\partial}{\partial u_{1}}+u_{21} \frac{\partial}{\partial u_{2}}+\cdots, \\
& D_{2}=\frac{\partial}{\partial x^{2}}+u_{2} \frac{\partial}{\partial u}+u_{12} \frac{\partial}{\partial u_{1}}+u_{22} \frac{\partial}{\partial u_{2}}+\cdots
\end{aligned}
$$

where

$$
u_{1}=\frac{\partial u}{\partial x^{1}}, \quad u_{11}=\frac{\partial^{2} u}{\partial\left(x^{1}\right)^{2}}, \quad u_{21}=\frac{\partial^{2} u}{\partial x^{2} \partial x^{1}}, \quad \ldots
$$

The operator $D_{1}$ and $D_{2}$ generate two commuting Taylor groups, whose finite transformation operators can be expressed by $T_{a}^{1}=e^{a D_{1}}$ and $T_{a}^{2}=e^{a D_{2}}$. Then two kinds of discrete shift operators can be written as

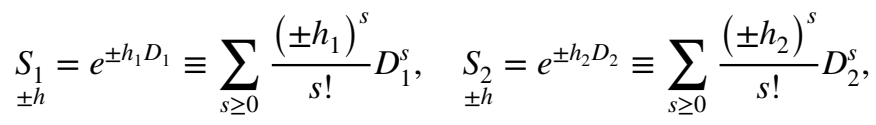

by fixing two arbitrary parameters $h_{i}>0, i=1,2$. Based on the definition of discrete shift operators, two pairs of discrete differentiation operators can be introduced as follows

$$
\begin{aligned}
& D_{i} \\
& \pm h
\end{aligned}= \pm \frac{1}{h_{i}}\left(\begin{array}{l}
S_{i}-1 \\
\pm h
\end{array}\right), \quad i=1,2
$$

Applying above operators to the variable $u$ to get the set of sequences $Z_{h}=\left(u_{1}, u_{11}, u_{12}, \ldots\right)$. Then one yields an enlarge space by combining $Z_{h}$ and $\left(x, u, u_{1}, u_{2}, \ldots\right)$, which can be denoted

$$
\tilde{Z}_{h}=\left(x, u, u_{1}, u_{2}, \cdots ; \underset{h}{u_{11}}, u_{12}, \cdots\right) .
$$

Proposition 1 If the condition is satisfied at each point $z \in \underset{h}{Z}$

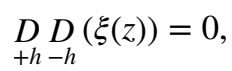

with $D$ and $D$ are right and left difference operators, the mesh $w$ remains uniform $\left(\tau_{+}^{*}=\tau_{-}^{*}, h_{+}^{*}=h_{-}^{*}\right)$ under the action of the transformation group $G_{1}$.

Proposition 2 The following condition

$$
\underset{ \pm h}{D}\left(\xi^{t}\right)=-\underset{ \pm \tau}{D}\left(\xi^{x}\right)
$$

is a necessary and sufficient condition to guarantee an orthogonal mesh $w$ to preserve its orthogonality in the plane $(t, x)$ under any transformation of the symmetry group $G_{1}$. 
We consider the case of two independent variables. $\underset{h}{Z}$ is the space of mesh variables $\left(t, x, u_{\tau}, u_{h}, \ldots, h^{+}, \tau^{+}\right)$. Then each finite-difference equation on the mesh $\underset{h}{w}$ can be expressed by

$$
E(z)=0,
$$

where $E \in A$ and $A$ is set of the analytic functions of finitely many coordinates $z^{i}$ of a vector $z \in{ }_{h}^{h}$. The finite-difference equation $E$ is written on finitely many points of the difference mesh $\underset{h}{w}$, which may be uniform or nonuniform. If the mesh is determined by the equation

$$
\Omega(z)=0,
$$

where $\Omega \in \underset{h}{A}$, the following proposition holds.

Proposition 3 A difference equation (3) written on the mesh (4) admits the transformation group $G_{1}$ with operator

$$
X=\xi^{t} \frac{\partial}{\partial t}+\xi^{x} \frac{\partial}{\partial x}+\eta \frac{\partial}{\partial u}+\cdots+h^{+} \underset{+h}{D}\left(\xi^{x}\right) \frac{\partial}{\partial h}+\tau_{+\tau}^{+} \underset{+\tau}{D}\left(\xi^{t}\right) \frac{\partial}{\partial \tau},
$$

it is necessary and sufficient that the following condition should be satisfied

$$
\left.X E(z)\right|_{(3),(4)}=0,\left.\quad X \Omega(z)\right|_{(3),(4)}=0 .
$$

For an evolution equation

$$
H\left(t, x, u, u_{x}, u_{t}, \ldots\right)=0,
$$

its potential systems are explicitly written as

$$
\begin{aligned}
& w_{t}=H_{1}\left(t, x, u, u_{x}, u_{t}, u_{x x} \cdots\right), \\
& w_{x}=H_{2}\left(t, x, u, u_{x}, u_{t}, u_{x x} \cdots\right),
\end{aligned}
$$

where $w_{t}$ is a potential variable. The potential system (8) can be reduced to equation (7) by means of the compatibility condition $w_{x t}=w_{t x}$. Based on the theory of Bluman's nonlocally related systems [24-26], we know that each local symmetry of potential system (8) projects onto a local symmetry of the original equation (7). Then the symmetry-preserving difference models can be constructed with the aid of potential system (8) of Eq. (7) and a fact presented in the following theorem.

Theorem 1 If the potential system of the finite-difference equation (3)

$$
\begin{gathered}
w_{x}=E_{1}, \\
h \\
w_{t}=-E_{2},
\end{gathered}
$$

where $\underset{+\tau}{D}\left(E_{1}\right)+\underset{+h}{D}\left(E_{2}\right)=E(z)$, admits the symmetries 


$$
X=\xi^{t} \frac{\partial}{\partial t}+\xi^{x} \frac{\partial}{\partial x}+\eta \frac{\partial}{\partial u}+\varphi \frac{\partial}{\partial w}
$$

then the difference model $E(z)$ admits the symmetries

$$
\bar{X}=\xi^{t} \frac{\partial}{\partial t}+\xi^{x} \frac{\partial}{\partial x}+\eta \frac{\partial}{\partial u}
$$

of original equation (7). In the continuous limit, the difference model $E(z)$ is changed to the evolution Eq. (7).

\section{Invariant Difference Model of the Benjamin-Ono Equation}

In this section, we consider the Benjamin-Ono equation

$$
u_{t t}+\alpha\left(u^{2}\right)_{x x}+\beta u_{x x x x}=0
$$

which describes the long internal gravity waves in deep stratified fluids [27]. The potential system of Eq. (9) can be written as

$$
w_{x}=u_{t}, \quad v_{x}=w, \quad v_{t}=-\alpha u^{2}-\beta u_{x x},
$$

where $w(x, t)$ and $v(x, t)$ are the potential variables. With the aid of Lie symmetry analysis, we can conclude that the potential system (10) admits the five-dimensional algebra of infinitesimal operators

$$
\begin{aligned}
& X_{1}=\frac{\partial}{\partial t}, X_{2}=\frac{\partial}{\partial x}, X_{3}=\frac{\partial}{\partial v}, X_{4}=x \frac{\partial}{\partial v}+\frac{\partial}{\partial w}, \\
& X_{5}=t \frac{\partial}{\partial t}+\frac{x}{2} \frac{\partial}{\partial x}-u \frac{\partial}{\partial u}-v \frac{\partial}{\partial v}-\frac{3 w}{2} \frac{\partial}{\partial w} .
\end{aligned}
$$

Substituting all the operators into conditions (1) and (2), we conclude that the mesh $w$ remain uniform and orthogonality in the plane $(t, x)$ under the action of the transformation group. It implies that the mesh equation has the form $h_{+}=h_{-}$, which satisfies the second condition in the Eq. (6). We consider the set of operators (11) in the space $\left(t, x, u, v, w, h, \tau, u_{-}, u_{+}, \hat{u}, v_{+}, \hat{v}, w_{+}\right)$. The corresponding stencil is shown in Fig. 1. It is obvious that the discrete subspace is thirteen-dimensional, $M \sim\left(t, x, u, v, w, h, \tau, u_{-}, u_{+}, \hat{u}, v_{+}, \hat{v}, w_{+}\right)$. The symmetry operators (11) prolonged to the difference stencil variables has the form

$$
\begin{aligned}
p r X= & \xi^{t} \frac{\partial}{\partial t}+\xi^{x} \frac{\partial}{\partial x}+\left(\hat{\xi}^{t}-\xi^{t}\right) \frac{\partial}{\partial \tau}+\left(\hat{\xi}^{x}-\xi^{x}\right) \frac{\partial}{\partial h}+\eta^{u} \frac{\partial}{\partial u}+\eta_{+}^{u} \frac{\partial}{\partial u_{+}}+\eta_{-}^{u} \frac{\partial}{\partial u_{-}} \\
& +\hat{\eta}^{u} \frac{\partial}{\partial \hat{u}}+\eta^{v} \frac{\partial}{\partial v}+\eta_{+}^{v} \frac{\partial}{\partial v_{+}}+\hat{\eta}^{v} \frac{\partial}{\partial \hat{v}}+\eta^{w} \frac{\partial}{\partial w}+\eta_{+}^{w} \frac{\partial}{\partial w_{+}},
\end{aligned}
$$




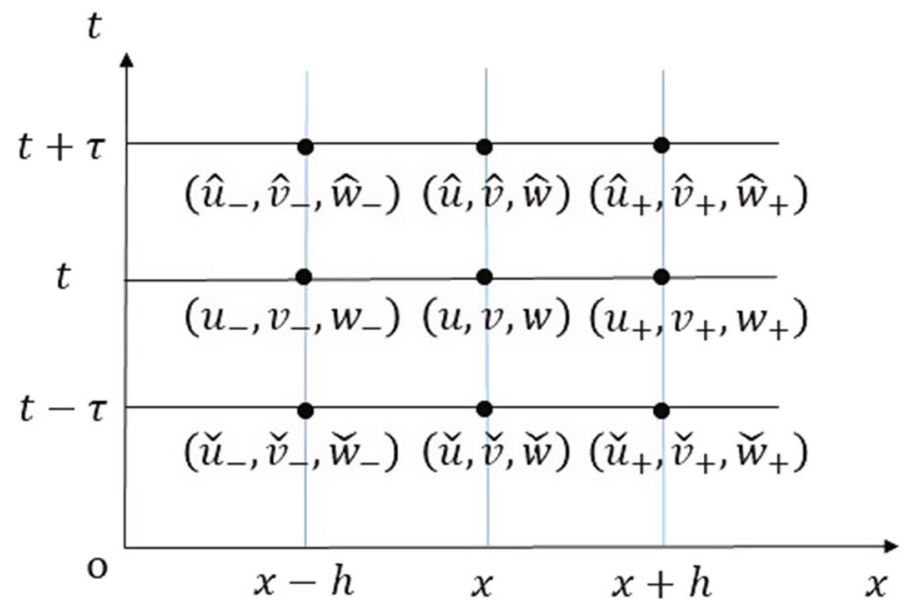

Fig. 1 The difference stencil of the Benjamin-Ono equation

where $\hat{f}=(t+\tau, x, u, v, w), \check{f}=(t-\tau, x, u, v, w)$, and $f_{ \pm}=(t, x \pm h, u, v, w)$. To find the finite-difference invariants $I_{j}$, one needs to solve the system of linear equations

$$
\operatorname{pr} X_{i}\left(t, x, u, v, w, h, \tau, u_{-}, u_{+}, \hat{u}, v_{+}, \hat{v}, w_{+}\right)=0, \quad i=1,2, \ldots, 5 .
$$

By solving above equations, we obtain eight invariants

$$
\begin{aligned}
& I_{1}=w_{+} h^{3}, \quad I_{2}=w h^{3}, \quad I_{3}=(\hat{u}-u) h^{2}, \quad I_{4}=\left(v_{+}-v\right) h^{2}, \\
& I_{5}=(\hat{v}-v) h^{2}, \quad I_{6}=\frac{h^{2}}{\tau}, \quad I_{7}=u h^{2}, \quad I_{8}=\left(u_{+}+u_{-}\right) h^{2} .
\end{aligned}
$$

By means of above invariants, we obtain following explicit scheme for Eq. (9)

$$
I_{1}-I_{2}=I_{3} I_{6}, \quad I_{4}=I_{2}, \quad I_{5} I_{6}=-\alpha I_{8}^{2}-\beta\left(I_{8}-2 I_{7}\right),
$$

which is is equivalent to

$$
w_{x}=u_{t}, \quad v_{x}=w, \quad v_{t}=-\alpha u^{2}-\beta u_{h} .
$$

Applying the difference operators $\underset{+\tau}{D, D} \underset{+h}{D}$ and compatibility

$$
u_{x t}=u_{t x}, \quad v_{h t}=v_{t x}, \quad w_{x t}=w_{t x},
$$

we obtain the invariant difference model for Eq. (9)

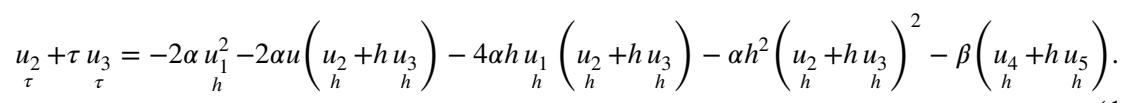


Theorem 2 The invariant difference model (13) preserves the symmetry operators (11).

Proof We extend the symmetry operators to the space $\left(t, x, u, v, w, \tau, h, u_{\tau}, u_{\tau}, u_{1}, u_{2}, u_{3}, u_{4}, u_{5}\right)$ as follows

$$
\begin{aligned}
\tilde{X}_{1}= & \frac{\partial}{\partial t}, \tilde{X}_{2}=\frac{\partial}{\partial x}, \tilde{X}_{3}=\frac{\partial}{\partial v}, \tilde{X}_{4}=x \frac{\partial}{\partial v}+\frac{\partial}{\partial w}, \\
\tilde{X}_{5}= & t \frac{\partial}{\partial t}+\frac{x}{2} \frac{\partial}{\partial x}-u \frac{\partial}{\partial u}-v \frac{\partial}{\partial v}-\frac{3 w}{2} \frac{\partial}{\partial w}+\tau \frac{\partial}{\partial \tau}+\frac{h}{2} \frac{\partial}{\partial h}-3 \underset{\tau}{u_{2}} \frac{\partial}{\partial u_{2}}-4 u_{\tau} \frac{\partial}{\partial u_{3}} \\
& -\frac{3}{2} u_{h} \frac{\partial}{\partial u_{1}}-2 u_{h} \frac{\partial}{\partial u_{2}}-\frac{5}{2} u_{h} \frac{\partial}{\partial u_{3}}-3 \underset{h}{u_{4}} \frac{\partial}{\partial u_{4}}-\frac{7}{2} u_{h} \frac{\partial}{\partial u_{5}} .
\end{aligned}
$$

Then we check the invariance condition (6) for the Eq. (13) and the operators (14)

$$
\left.\tilde{X}_{1}(\Lambda)\right|_{(13)}=0,\left.\tilde{X}_{2}(\Lambda)\right|_{(13)}=0,\left.\tilde{X}_{3}(\Lambda)\right|_{(13)}=0,\left.\tilde{X}_{4}(\Lambda)\right|_{(13)}=0,\left.\tilde{X}_{5}(\Lambda)\right|_{(13)}=0 \text {, }
$$

where $\Lambda=\underset{\tau}{u_{2}}+\tau u_{\tau}+2 \alpha u_{1}^{2}+2 \alpha u\left(\begin{array}{c}u_{2}+h u_{3} \\ h\end{array}\right)+4 \alpha h u_{1}\left(\begin{array}{c}u_{2}+h u_{3} \\ h\end{array}\right)+\alpha h^{2}\left(\begin{array}{c}u_{2}+h u_{3} \\ h\end{array}\right)^{2}$ $+\beta\left(\begin{array}{cc}u_{4}+h u_{5} \\ h\end{array}\right)^{\tau}$. Thus we prove that the difference model (13) admits the symmetry group with operators (11), which means the difference model preserves original continuous symmetries.

\section{Invariant Difference Model of the Nonlinear Dispersive $K(m, n)$ Equation}

\subsection{Invariant Difference Model of the Nonlinear Dispersive $K(2,3)$ Equation}

In 1993, Rosenau and Hyman [28] derived a family of fully nonlinear KdV equations which is called $K(m, n)$ equation

$$
u_{t}+\left(u^{m}\right)_{x}+\left(u^{n}\right)_{x x x}=0, \quad m>0,1<n \leq 3 .
$$

The physical phenomenon described by this equation can explain the nonlinear dispersion can compactify solitary waves and generate compactons. When $m=n=2$ or 3, Eq. (15) is reduced to the $K(2,2)$ and the $K(3,3)$ equation [29]. Firstly, we consider nonlinear dispersive $K(m, n)$ equation as $m=2$ and $n=3$.

$$
u_{t}+\left(u^{2}\right)_{x}+\left(u^{3}\right)_{x x x}=0 .
$$

The potential system of Eq. (16) can be written as 


$$
w_{x}=u, \quad w_{t}=-u^{2}-3 u^{2} u_{x x}-6 u u_{x}^{2} .
$$

We can conclude that this potential system possesses following four symmetry operators

$$
X_{1}=\frac{\partial}{\partial t}, X_{2}=\frac{\partial}{\partial x}, X_{3}=\frac{\partial}{\partial w}, X_{4}=-\frac{t}{2} \frac{\partial}{\partial t}+\frac{x}{2} \frac{\partial}{\partial x}+u \frac{\partial}{\partial u}+\frac{3 w}{2} \frac{\partial}{\partial w},
$$

through Lie symmetry analysis. All the operators of (17) satisfy conditions (1) and (2). Thus, one can directly utilize the orthogonal gride which is uniform in $t$ and $x$ directions. We consider the set of operators (17) in the space $\left(t, x, u, w, h, \tau, u_{-}, u_{+}, w_{+}, \hat{w}\right)$. The corresponding stencil is shown in Fig. 2. In this case, the discrete subspace is ten-dimensional, $M \sim\left(t, x, u, w, h, \tau, u_{-}, u_{+}, w_{+}, \hat{w}\right)$. By solving the characteristic equations of the prolonged symmetry, one can obtain following six difference invariants

$$
I_{1}=\frac{u}{h^{2}}, \quad I_{2}=\frac{u_{+}}{h^{2}}, \quad I_{3}=\frac{u_{-}}{h^{2}}, \quad I_{4}=\tau h, \quad I_{5}=\frac{w_{+}-w}{h^{3}}, \quad I_{6}=\frac{\hat{w}-w}{h^{3}} .
$$

By means of above invariants, the scheme for Eq. (16) can be written as

$$
I_{5}=I_{1}, \quad I_{6}=-I_{1}^{2} I_{4}-3 I_{1}^{2} I_{4}\left[\left(I_{2}-I_{1}\right)-\left(I_{1}-I_{3}\right)\right]-6 I_{1} I_{4}\left(I_{2}-I_{1}\right)\left(I_{1}-I_{3}\right),
$$

which is is equivalent to

$$
w_{x}=u, \quad w_{t}=-u^{2}-3 u^{2} u_{h}-6 u u_{h}\left(\begin{array}{cr}
u_{1}-h u_{2} \\
h
\end{array}\right) .
$$

Applying the difference operators $\underset{+\tau}{\underset{\tau}{D}}$ and $\underset{+h}{D}$ to Eqs. (18) respectively, one obtains the invariant difference model for Eq.(16)

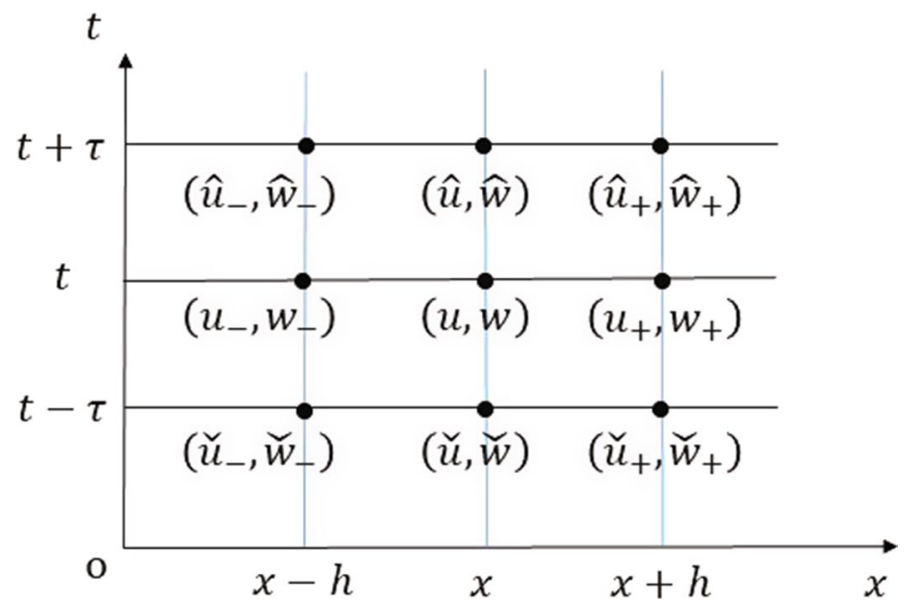

Fig. 2 The difference stencil of the nonlinear dispersive $K(2,3)$ equation 


$$
u_{t}=-2 u u_{h}-h u_{1}^{2}-3 u^{2} u_{h}-6 \underset{h}{u_{1}^{3}}+6 h u_{h}^{2} u_{h}-18 u u_{h} u_{h} u_{2}-15 h u_{h}^{2} u_{h}-12 h u \underset{h}{u_{1}} u_{h}-9 h^{2} u_{1}^{2} u_{3} .
$$

Theorem 3 The invariant difference model (19) preserves the symmetry operators (17).

Proof The symmetry operators extended to the space $\left(t, x, u, w, \tau, h, \underset{\tau}{2}, u_{1}, u_{2}, u_{3}\right)$ can be written as

$$
\begin{aligned}
& \tilde{X}_{1}=\frac{\partial}{\partial t}, \quad \tilde{X}_{2}=\frac{\partial}{\partial x}, \quad \tilde{X}_{3}=\frac{\partial}{\partial w}, \\
& \tilde{X}_{4}=-\frac{t}{2} \frac{\partial}{\partial t}+\frac{x}{2} \frac{\partial}{\partial x}+u \frac{\partial}{\partial u}+\frac{3 w}{2} \frac{\partial}{\partial w}-\frac{\tau}{2} \frac{\partial}{\partial \tau}+\frac{h}{2} \frac{\partial}{\partial h}+\frac{3}{2} u_{\tau} \frac{\partial}{\partial u_{t}}+\frac{1}{2} u_{h} \frac{\partial}{\partial u_{1}}-\frac{1}{2} u_{h} \frac{\partial}{\partial u_{3}} .
\end{aligned}
$$

Then we check the invariance condition (6) for the Eq. (19) and the operators (17)

$$
\left.\tilde{X}_{1}(\Psi)\right|_{(19)}=0,\left.\tilde{X}_{2}(\Psi)\right|_{(19)}=0,\left.\tilde{X}_{3}(\Psi)\right|_{(19)}=0,\left.\tilde{X}_{4}(\Psi)\right|_{(19)}=0,
$$

where $\Psi=u_{t}+2 u u_{1}+h u_{h}^{2}+3 u_{h}^{2} u_{3}+6 u_{h}^{3}-6 h u_{h}^{2} u_{2}+18 u u_{h} u_{h} u_{2}+15 h u_{1}^{2} u_{2}+12 h u u_{h} u_{h}+9 h^{2} u_{1}^{2} u_{3}$. After checking above identical equations, we can conclude that the difference equation (19) admits the symmetry group with operators (17), which means the difference model preserves original continuous symmetries.

Remark 1 If Eq. (15) admits the compactons in the classical sense, the upper limit on $n \leq 3$ is necessary [28]. Rosenau and Hyman had studied four cases $m, n=2,3$, and they have proved that the $K(2,2), K(2,3), K(3,2)$ and $K(3,3)$ equations all possess compactons. It is a fact that all the four equations are not integrable. Heredero et al. had studied the integrability of the full $K(m, n)$ family of equations from the perspective of the formal symmetries [30, 31]. Based on the technique of Painlevé analysis, we know all the four cases do not possess the Painlevé property [32]. For the $K(2,2)$ equation, its potential system can be written as

$$
\begin{aligned}
& w_{x}=u, \\
& w_{t}=-\left(u^{2}+2 u_{x}^{2}+2 u u_{x x}\right) .
\end{aligned}
$$

The Lie point symmetries of this potential system consist of $X_{1}=\frac{\partial}{\partial x}, X_{2}=\frac{\partial}{\partial t}, X_{3}=\frac{\partial}{\partial w}$ and $X_{4}=-t \frac{\partial}{\partial t}+u \frac{\partial}{\partial t}+w \frac{\partial}{\partial w}$. Since all the symmetries can not be extended the operators containing both difference stencil variables $h$ and $\tau$, these four symmetries can not be used to construct the symmetry-preserving difference models for $K(2,2)$ equation. $K(3,3)$ equation and $K(2,2)$ equation have similar symmetric structure, so it is impossible to construct symmetry-preserving difference model for the same reason. 


\subsection{Invariant Difference Model of the Nonlinear Dispersive $K(3,2)$ Equation}

If one takes $m=3$ and $n=2$ in Eq. (15), the $K(3,2)$ equation can be obtained as

$$
u_{t}+\left(u^{3}\right)_{x}+\left(u^{2}\right)_{x x x}=0 .
$$

The potential system of the $K(3,2)$ equation can be constructed as follows

$$
\begin{aligned}
& w_{x}=u, \\
& w_{t}=-\left(u^{3}+2 u_{x}^{2}+2 u u_{x x}\right) .
\end{aligned}
$$

We can obtained the following four symmetry operators

$$
X_{1}=\frac{\partial}{\partial x}, \quad X_{2}=\frac{\partial}{\partial t}, \quad X_{3}=\frac{\partial}{\partial w}, X_{4}=-5 t \frac{\partial}{\partial t}-x \frac{\partial}{\partial x}+2 u \frac{\partial}{\partial u}+w \frac{\partial}{\partial w} .
$$

By utilizing the orthogonal gride presented in Fig. 2, six difference invariants can be obtained as

$$
\begin{aligned}
& I_{1}=u h^{2}, \quad I_{2}=u_{+} h^{2}, \quad I_{3}=u_{-} h^{2}, \quad I_{4}=\frac{\tau}{h^{5}}, \\
& I_{5}=\left(w_{+}-w\right) h, \quad I_{6}=(\hat{w}-w) h,
\end{aligned}
$$

Then the scheme for $K(3,2)$ equation can be established as following form

$$
I_{5}=I_{1}, I_{6}=-I_{1}^{2} I_{4}-2 I_{4}\left(I_{2}-I_{1}\right)\left(I_{1}-I_{3}\right)-2 I_{1} I_{4}\left[\left(I_{2}-I_{1}\right)-\left(I_{1}-I_{3}\right)\right],
$$

which has the equivalent form

$$
w_{x}=u, \quad w_{t}=-u^{3}-2 \underset{h}{u_{1}}\left(\begin{array}{cc}
u_{1}-h u_{2} \\
h
\end{array}\right)-2 u u_{2} .
$$

Applying the compatibility of Eq. (22), one yields the invariant difference model

$$
u_{t}=-3 u^{2} u_{1}-3 h u u_{1}^{2}-h^{2} u_{1}^{3}-6 \underset{h}{u_{1}} u_{2}+2 h u_{2}^{2}-2 u \underset{h}{u_{3}}-2 h u_{1} u_{3} .
$$

Based on the analysis of Theorems 2 and 3, we can conclude that the difference model (23) preserves all the symmetries.

\section{Invariant Difference Model of the Hunter-Saxton Equation}

Hunter-Saxton equation is firstly derived by Hunter and Saxton to describe the weakly nonlinear hyperbolic waves governed by variational principles [33]. In 2014, Hou et al. [34] derived the Hunter-Saxton hierarchy, in which the first equation is given as

$$
u_{x x t}+4 u_{x} u_{x x}+2 u u_{x x x}=0
$$

Furthermore, the Hunter-Saxton can be seen as the high frequency limit of the Camassa-Holm equation [35-37] 


$$
u_{t}-u_{x x t}+3 u u_{x}-2 u_{x} u_{x x}-u u_{x x x}=0 .
$$

The potential system of Eq. (24) can be written as

$$
w_{x}=u_{x x}, \quad w_{t}=-\left(2 u u_{x x}+u_{x}^{2}\right) .
$$

Base on the theory of symmetry analysis, the Lie algebra of Eq. (24) can be expanded by following six symmetry operators

$$
\begin{aligned}
& X_{1}=\frac{\partial}{\partial x}, \quad X_{2}=\frac{\partial}{\partial t}, \quad X_{3}=\frac{\partial}{\partial v}, \quad X_{4}=x \frac{\partial}{\partial x}+u \frac{\partial}{\partial u}, \\
& X_{5}=-t \frac{\partial}{\partial t}-x \frac{\partial}{\partial x}+w \frac{\partial}{\partial w}, \quad X_{6}=-f^{\prime}(t) \frac{\partial}{\partial u}+2 f(t) \frac{\partial}{\partial x} .
\end{aligned}
$$

The operators $X_{4}, X_{5}$ and $X_{6}$ do not contain all the variable about $x, t, u$ and $w$, thus the operators can not be expanded to the space $\left(t, x, u, w, h, \tau, u_{-}, u_{+}, w_{+}, \hat{w}\right)$ to construct the invariant difference model. However, the linear combination of $X_{4}$ and $X_{5}$ contains all the variables. What follows, we shall consider the operators $\lambda X_{4}+X_{5}$ $(\lambda \neq 1)$, which has the form

$$
-t \frac{\partial}{\partial t}+(\lambda-1) x \frac{\partial}{\partial x}+\lambda u \frac{\partial}{\partial u}+w \frac{\partial}{\partial w} .
$$

By solving the characteristic equations of the extended form of above symmetry, the six difference invariants can be obtained

$$
\begin{aligned}
& I_{1}=u h^{-\frac{\lambda}{\lambda-1}}, \quad I_{2}=u_{+} h^{-\frac{\lambda}{\lambda-1}}, \quad I_{3}=u_{-} h^{-\frac{\lambda}{\lambda-1}}, \quad I_{4}=\tau h^{\frac{1}{\lambda-1}}, \\
& I_{5}=\left(w_{+}-w\right) h^{-\frac{1}{\lambda-1}}, \quad I_{6}=(\hat{w}-w) h^{-\frac{1}{\lambda-1}} .
\end{aligned}
$$

By means of above invariants, the scheme for Eq. (24) can be written as

$$
I_{5}=\left(I_{2}-I_{1}\right)-\left(I_{1}-I_{3}\right), \quad I_{6}=I_{4}\left(I_{2}-I_{1}\right)\left(I_{1}-I_{3}\right)-2 I_{1} I_{4}\left[\left(I_{2}-I_{1}\right)-\left(I_{1}-I_{3}\right)\right] .
$$

The equivalent form of this scheme is

$$
w_{h}=\underset{h}{u_{2},} \quad w_{t}=-2 u u_{2}-\underset{h}{u_{1}}\left(\begin{array}{c}
u_{1}-h u_{2} \\
h
\end{array}\right) .
$$

Applying the difference operators $\underset{+\tau}{D}$ and $\underset{+h}{D}$ to above two equations respectively, one has the following invariant difference model

$$
u_{112}=-4 u_{h} u_{h}-3 h u_{1} u_{h}-2 u u_{h}+h u_{h}^{2}+2 h^{2} u_{2} u_{h}+h^{3} u_{3}^{2}-h u_{2}-h^{2} u_{3},
$$

where $u_{112}=\underset{+\tau+h+h}{D} \underset{h}{D} D(u)$. The difference model preserves the symmetry operators

$$
X_{1}, X_{2}, X_{3}, \lambda X_{4}+X_{5} .
$$


Remark 2 This section gives an example to illustrate the scheme presented in this paper can be used to construct the invariant difference model not only preserving all the symmetries, but also maintaining some linear combination of the symmetries. This idea inspires us to study some other types of integrable systems to investigate the invariant difference models to further study the numerical solutions. For the Camassa-Holm equation, it has the symmetry operators $X_{1}=\frac{\partial}{\partial t}, X_{2}=\frac{\partial}{\partial x}$ and $X_{3}=-t \frac{\partial}{\partial t}+u \frac{\partial}{\partial u}$. The symmetries of Camassa-Holm equation are consistent with $K(2,2)$ equation. Therefore, these symmetries can not be employed to construct invariant difference model.

\section{Conclusions}

In this paper, we focus on investigating the symmetry invariant difference models of high order continuous differential equations. The procedure of constructing symmetry-preserving difference meshes with the aid of potential systems of original equations is briefly introduced. Then the potential systems of the Benjamin-Ono equation and the nonlinear dispersive $K(m, n)$ equation are employed to construct the difference models inherited the symmetries of the original equations. The symmetry invariance of the obtained difference models are also checked by considering the symmetries extended to the difference variables. For the $K(m, n)$ equation, we consider four cases which yield compactons, i.e. $m, n=2,3$. It is proved that one can construct the invariant difference models preserving all the symmetries when $m \neq n$. In order to further expand the method of this paper, other types of equations are studied. The symmetries of these equations can not be used to construct difference invariants, but the linear combinations of partial symmetries can be used to construct difference invariants. The Hunter-Saxton equation is investigated as an example. The idea of this paper can be further utilized to investigate the symmetrypreserving difference models of some other types of integrable equations.

Acknowledgements The author would like to express his thanks to the editor and reviewers for their valuable suggestions and comments. This work is supported by Shanxi Province Science Foundation for Youths (No. 201901D211274), Research Project Supported by Shanxi Scholarship Council of China (No. 2020-105), Scientific and Technological Innovation Programs of Higher Education Institutions in Shanxi (No. 2019L0531) and the Fund for Shanxi "1331KIRT”.

\section{Declarations}

Conflict of interest The authors declare they have no conflicts of interest.

Open Access This article is licensed under a Creative Commons Attribution 4.0 International License, which permits use, sharing, adaptation, distribution and reproduction in any medium or format, as long as you give appropriate credit to the original author(s) and the source, provide a link to the Creative Commons licence, and indicate if changes were made. The images or other third party material in this article are included in the article's Creative Commons licence, unless indicated otherwise in a credit line to the material. If material is not included in the article's Creative Commons licence and your intended use is not permitted by statutory regulation or exceeds the permitted use, you will need to obtain permission directly from the copyright holder. To view a copy of this licence, visit http://creativecommons.org/licen ses/by/4.0/. 


\section{References}

1. Bluman, G..W., Anco, S..C.: Symmetry and Itegration Methods for Differential Equations. Springer (2002)

2. Bluman, G..W., Cheviakov, A..F., Anco, S..C.: Applications of Symmetry Methods to Partial Differential Equations. Springer (2010)

3. Zhao, Z.L.: Bäcklund transformations, rational solutions and soliton-cnoidal wave solutions of the modified Kadomtsev-Petviashvili equation. Appl. Math. Lett. 89, 103-110 (2019)

4. Zhao, Z.L., Han, B.: Residual symmetry, Bäcklund transformation and CRE solvability of a (2+1)-dimensional nonlinear system. Nonlinear Dyn. 94(1), 461-474 (2018)

5. Zhao, Z.L., Han, B.: Lie symmetry analysis, Bäcklund transformations, and exact solutions of a (2+1)-dimensional Boiti-Leon-Pempinelli system. J. Math. Phys. 58(10), 101514 (2017)

6. Zhao, Z.L., He, L.C.: Lie symmetry, nonlocal symmetry analysis, and interaction of solutions of a (2+1)-dimensional KdV-mKdV equation. Theor. Math. Phys. 206(2), 142-162 (2021)

7. Lu, H.H., Zhang, Y.F.: Some generalized isospectral-nonisospectral integrable hierarchies. Commun. Nonlinear Sci. Numer. Simul. 100, 105851 (2021)

8. Tian, S.F.: Lie symmetry analysis, conservation laws and solitary wave solutions to a fourth-order nonlinear generalized Boussinesq water wave equation. Appl. Math. Lett. 100, 106056 (2020)

9. Zhang, Y.F., Mei, J.Q., Guan, H.Y.: A method for generating isospectral and nonisospectral hierarchies of equations as well as symmetries. J. Geom. Phys. 147, 103538 (2020)

10. Dong, M.J., Tian, S.F., Yan, X.W., Zhang, T.T.: Nonlocal symmetries, conservation laws and interaction solutions for the classical Boussinesq-Burgers equation. Nonlinear Dyn. 95, 273-291 (2019)

11. Maeda, S.: The similarity method for difference equations. IMA J. Appl. Math. 38(2), 129-134 (1987)

12. Levi, D., Winternitz, P.: Symmetries and conditional symmetries of differential-difference equations. J. Math. Phys. 34(8), 3713-3730 (1993)

13. Shen, S.F., Qu, C.Z.: Symmetry groups of differential-difference equations and their compatibility. J. Math. Anal. Appl. 371(1), 355-362 (2010)

14. Ye, C.E., Huang, Q., Shen, S.F., Jin, Y.Y.: A symmetry classification algorithm of the generalized differential-difference equations. Appl. Math. Lett. 74, 27-32 (2017)

15. Sahadevan, R., Khousalya, S., Nalini Devi, L.: Nonlocal symmetries and recursion operators: partial differential and differential-difference equations. J. Math. Anal. Appl. 308(2), 636-655 (2005)

16. Dorodntisyn, V.A.: Finite difference models entirely inheriting continuous symmetry of original differential equations. Int. J. Mod. Phys. C 05(04), 723-734 (1994)

17. Dorodnitsyn, V., Winternitz, P.: Lie point symmetry preserving discretizations for variable coefficient Korteweg-de Vries equations. Nonlinear Dyn. 22(1), 49-59 (2000)

18. Budd, C., Dorodnitsyn, V.: Symmetry-adapted moving mesh schemes for the nonlinear schrödinger equation. J. Phys. A Math. Gen. 34(48), 10387-10400 (2001)

19. Xin, X.P., Chen, Y.: The using of conservation laws in symmetry-preserving difference scheme. Commun. Theor. Phys. 59(5), 573-578 (2013)

20. Xin, X.P., Chen, Y., Wang, Y.H.: A symmetry-preserving difference scheme for high dimensional nonlinear evolution equations. Chin. Phys. B 22(6), 060201 (2013)

21. Ma, P.L., Tian, S.F., Zhang, T.T.: On symmetry-preserving difference scheme to a generalized Benjamin equation and third-order Burgers equation. Appl. Math. Lett. 50, 146-152 (2015)

22. Zhang, T.T., Xu, M.J.: The symmetry-preserving difference schemes and exact solutions of some high-dimensional differential equations. Appl. Math. Lett. 112, 106813 (2021)

23. Dorodnitsyn, V.: Applications of Lie Groups to Difference Equations. CRC Press (2011)

24. Bluman, G.W., Cheviakov, A.F.: Framework for potential systems and nonlocal symmetries: algorithmic approach. J. Math. Phys. 46(12), 123506 (2005)

25. Bluman, G.W., Cheviakov, A.F., Ivanova, N.M.: Framework for nonlocally related partial differential equation systems and nonlocal symmetries: extension, simplification, and examples. J. Math. Phys. 47(11), 113505 (2006)

26. Zhao, Z.L.: Conservation laws and nonlocally related systems of the Hunter-Saxton equation for liquid crystal. Anal. Math. Phys. 9(4), 2311-2327 (2019)

27. Li, Y.Y., Hu, H.C.: Nonlocal symmetries and interaction solutions of the Benjamin-Ono equation. Appl. Math. Lett. 75, 18-23 (2018) 
28. Rosenau, P., Hyman, J.M.: Compactons: Solitons with finite wavelength. Phys. Rev. Lett. 70, 564567 (1993)

29. Wazwaz, A.M.: New solitary-wave special solutions with compact support for the nonlinear dispersive K(m, n) equations. Chaos Solitons Fractals 13(2), 321-330 (2002)

30. Heredero, R.H., Euler, M., Euler, N., Reyes, E.G.: Compacton equations and integrability: the Rosenau-Hyman and Cooper-Shepard-Sodano equations. Discrete Contin. Dyn. Syst. 40, 529-548 (2020)

31. Heredero, R.H., Reyes, E.G.: Nonlocal symmetries, compacton equations, and integrability. Int. J. Geom. Methods Mod. Phys. 10(9), 1350046 (2013)

32. Banerjee, R.S.: Painlevé analysis of the $\mathrm{K}(\mathrm{m}, \mathrm{n})$ equations which yield compactons. Phys. Scr. 57, 598-600 (1998)

33. Hunter, J.K., Saxton, R.: Dynamics of director fields. SIAM J. Appl. Math. 51(6), 1498-1521 (1991)

34. Hou, Y., Fan, E.G., Zhao, P.: Algebro-geometric solutions for the Hunter-Saxton hierarchy. Zeitschrift für angewandte Mathematik und Physik 65, 487-520 (2014)

35. Fuchssteiner, B., Fokas, A.S.: Symplectic structures, their Bäcklund transformations and hereditary symmetries. Phys. D Nonlinear Phenom. 4(1), 47-66 (1981)

36. Camassa, R., Holm, D.D.: An integrable shallow water equation with peaked solitons. Phys. Rev. Lett. 71(11), 1661-1664 (1993)

37. Camassa, R., Holm, D.D., Hyman, J.M.: A new integrable shallow water equation. Adv. Appl. Mech. 31, 1-33 (1994) 